\title{
A multi-factor interaction model (MFIM) for damage initiation and progression
}

\author{
C. C. Chamis \\ NASA Glenn Research Center Cleveland, USA
}

\begin{abstract}
A Multi-Factor-Interaction-Model (MFIM) is briefly described to represent complex point material behavior in a single equation. The model is of product form in order to represent coupled interactions and to be computationally effective. The model describes a continuum or surface in space that represents the complex material behavior in terms of the various factors that affect a specified material behavior. The material specified behavior is inclusive of all material properties, mechanical, thermal, physical and effects thereon, such as temperature, time, cyclic loadings, etc. Sample case results simulated by using MFIM are compared with test data to illustrate its versatility and its relevance to reality. These results show that the MFIM can accurately predict metal matrix composite fatigue data and mechanical properties of a steel alloy. Helpful guidelines for its effective use are also included.
\end{abstract}

Keywords: material properties, high temperature, nonlinearities.

\section{Introduction}

The simulation of complex material behavior resulting from the interaction of several factors (such as temperature, nonlinear material due to high stress, time dependence, fatigue, etc), has been mainly performed by factor-specific representations. For example, entire text books are devoted to plasticity, creep, fatigue and high strain rate to mention only a few. Investigators have derived equations that describe material behavior for each factor-specific effect. Suppose we visualize that the material behavior is a continuum represented by some surface. Then, we can think of some representation which describes that surface which is inclusive of all participating factors that affect material behavior either singly or interactively in various combinations. To that end, research has 
been a continuing activity at Glenn Research Center for about twenty-five years. It started with a primitive form of MFIM representation for describing complex composite behavior in polymer matrix composites [1]. It was extended to metal matrix composites [2] and continues to be evolved in Aerospace Plane and the High Speed support of the National Research Programs. The result of all this research is that general guidelines for its usage are briefly described. Simulation results are presented and compared (where available) with experimental data to illustrate its versatility and perhaps demonstrate the claim for its uniqueness.

\section{Fundamental considerations and the (MFIM)}

We start with the premise that if we are to quantify the range of factors affecting material properties, we need a description of material behavior. In this context, it is reasonable to consider that material behavior constitutes an n-dimensional space (Material Behavior Space (MBS)) where each point represents a specific aspect of material behavior. It is further reasonable to assume that MBS can be described by an assumed interpolation function. One convenient interpolation function is a polynomial of product form because mutual interactions among different factors can be represented by the overall product, and includes those cross products in common algebraic polynomials. In this investigation, MBS is assumed to be described by the following multifactor interaction equation (MFIM):

$$
\frac{M_{P}}{M_{P_{0}}}=\prod_{i=1}^{N} A_{i}^{m_{i}}
$$

where $\mathrm{M}_{\mathrm{P}}$ is the property affected to be evaluated. $\mathrm{M}_{\mathrm{Po}}$ corresponds to the initial (reference) material state or condition. $\mathrm{A}_{\mathrm{i}}$ represents the ith factor that influences material behavior, and $\mathrm{m}_{\mathrm{i}}$ is an exponent. $\mathrm{A}_{\mathrm{i}}$ is further defined by:

$$
A_{i}=\left(1-\frac{B}{B_{0}}\right)
$$

Here B represents a specific cause factor for behavior (for example, temperature), and $B_{o}$ is the corresponding final value. This concept is schematically represented in Fig. 1, the development of the multi-factorinteraction model (MFIM) to represent complex material behavior by a single equation. The objective of the present paper is to briefly describe MFIM and present results obtained there from to illustrate its uniqueness and its versatility.

Values for $B_{o}$ and $m_{i}$ for specific behavior are selected either from known behavior or more likely from a best judgment in conjunction with consultations with seasoned professionals for that behavior. By representing the MBS with the MFIM of product form (Eq. (1)), we gain another distinct advantage. The behavior factors, B, can also be represented by another level of MFIM or progressive substructuring of equation (1). The progressive substructuring leads to a multitier representation of the MBS that permits intrinsic lower tier behaviors to influence more than one factor at the next higher tier. In other words, the observed specific behavior $\left(\mathrm{B}_{\mathrm{i}}\right.$ may depend on another set of lower 
tier elemental behaviors). Further, the behavior factors in this lower set of specific behaviors may depend on yet another next lower tier of elemental behaviors. That is, there are usually sets and subsets of specific behaviors that hierarchically influence the higher level behaviors. When this is done, $\mathrm{N}$ can be limited to 6, (for example), but the number of factors influencing material behavior at the next lower tier will increase exponentially as $\mathrm{N}^{\mathrm{j}}$ where $\mathrm{j}$ is the number of 6-factor tiers. For example, when $\mathrm{j}=3, \mathrm{~N}=216$, and so forth. This representation is natural for multiparallel processing computers where the tiers are programmed with different granularities. Obviously, then, the motivation for selecting such a form is for computational and programming effectiveness. Another reason for selecting an MFIM of product form is that the effect of each factor can be evaluated separately. The interpretation of $\mathrm{B}_{0}$ is that it represents a scale, whereas $m_{i}$ represents a shape or path. For example, $\left(1-B / B_{0}\right)^{m}{ }_{i}$ where 1 $>\mathrm{B} / \mathrm{B}_{0}$ and $+\infty<\mathrm{m}_{\mathrm{i}}<-\infty$, covers the whole space as is illustrated in Fig. 2 .

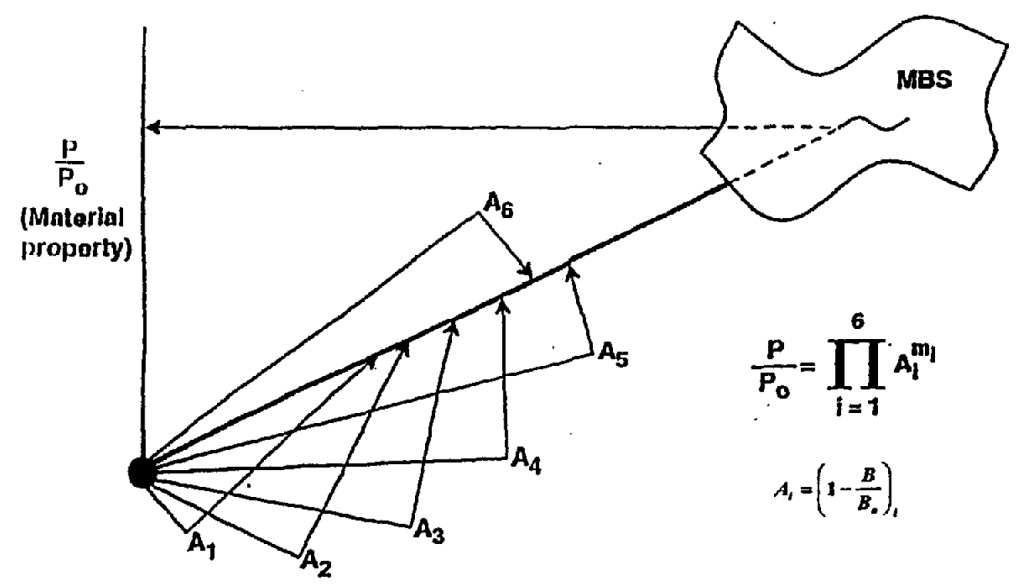

Factors influencing material behavior ,

Figure 1: Conceptual schematic of material behaviour through a MultiFactor Interaction Model (MFIM).

The inclusiveness of this particular form, combined with its simplicity, makes it very attractive for a computational simulation. An expanded form of MFIM is shown in Eq. (3) below:

$$
\begin{aligned}
\frac{M p}{M p o}= & \left(\frac{T_{g w}-T}{T_{g w}-T_{o}}\right)^{m}\left(1-\frac{\boldsymbol{\sigma}}{S_{f}}\right)^{n}\left(1-\frac{\boldsymbol{\sigma} t}{S_{f} t_{f}}\right)^{p}\left(1-\frac{\boldsymbol{\sigma}_{M} N_{M}}{S_{f} N_{f M}}\right)^{q}\left(1-\frac{\boldsymbol{\sigma}_{T} N_{T}}{S_{f} N_{f T}}\right)^{r}\left(1-\frac{\boldsymbol{\omega}}{\boldsymbol{\omega}_{f}}\right)^{s} \ldots \\
& \ldots\left(1-\frac{E_{e}}{E_{e f}}\right)^{u}\left(1-\frac{C_{e}}{C_{e f}}\right)^{v}\left(1-\frac{C_{c}}{C_{c f}}\right)^{w} \ldots
\end{aligned}
$$

The Multifactor Equation 
for inclusiveness and convenience of presentation. The top line includes six factors in parentheses: $\left(\mathrm{T}_{\mathrm{gw}}-\mathrm{T}\right) /\left(\mathrm{T}_{\mathrm{gw}}-\mathrm{T}_{0}\right)$ denotes temperature effects: $(1-$ $\left.\sigma / \mathrm{S}_{\mathrm{f}}\right)$ denotes combined stress effect; $\left(1-\sigma \mathrm{t} / \mathrm{S}_{\mathrm{f}} \mathrm{t}_{\mathrm{f}}\right)$ denotes time dependent effects: $\left(1-\sigma_{\mathrm{m}} \mathrm{N}_{\mathrm{m}} / \mathrm{S}_{\mathrm{f}} \mathrm{N}_{\mathrm{fm}}\right)$ denotes mechanical cyclic load effects where $\sigma_{\mathrm{m}}$ is the cyclic stress due to $\mathrm{N}_{\mathrm{M}}$ cycling; $\left(1-\sigma_{\mathrm{T}} \mathrm{N}_{\mathrm{T}} / \mathrm{S}_{\mathrm{f}} \mathrm{N}_{\mathrm{ft}}\right)$ denotes thermal stress due to $\mathrm{N}_{\mathrm{T}}$ cycling; $\left(1-\omega / \omega_{\mathrm{f}}\right)$ denotes frequency effects due to $\omega-$ frequency. The factors in the second line denote erosion, corrosion and chemical or metallurgical effects. The dots between the first and second lines indicate that several other factors can be included. Suffice it to say that the MFIM is generic and inclusive. Two points to be noted are: (1) not all terms have to be included; and (2) substructuring may be appropriate after six factors. For example, the three factors in the second line affect some, if not all the factors in the first line. This definitely is the case for the chemical/metallurgical factor.

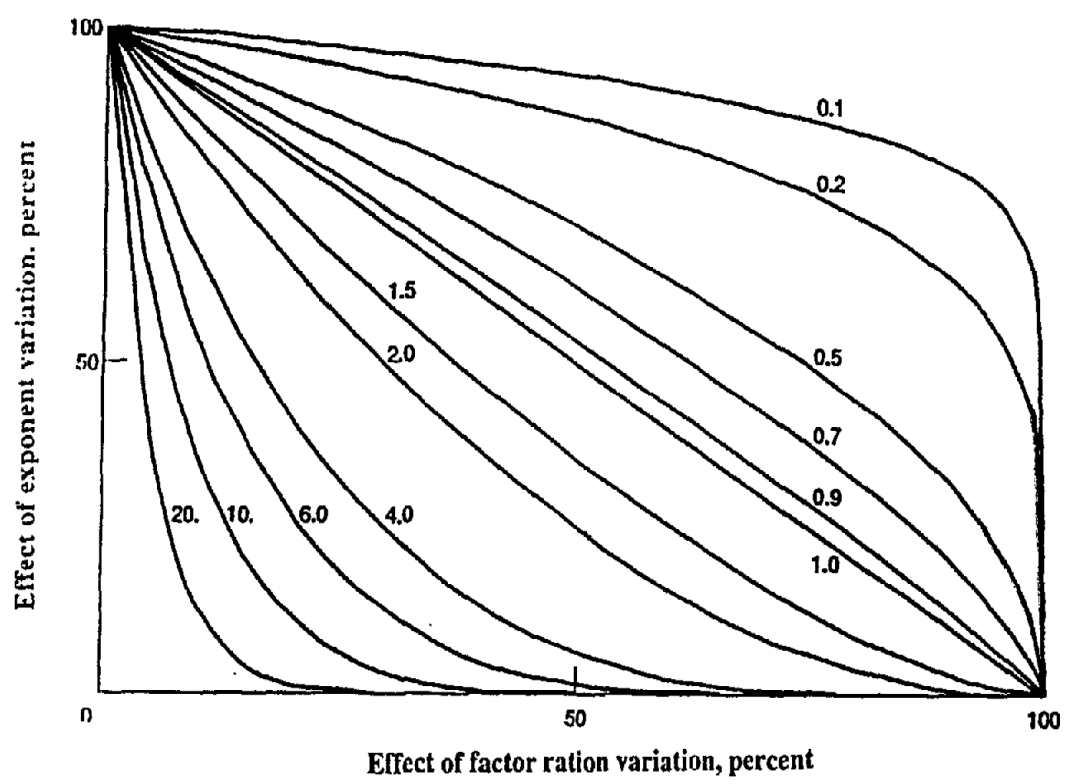

Figure 2: Effect of variation in the exponent.

\section{Guidelines for usage of the (MFIM)}

Some general guidelines for usage of the MFIM are appropriate: (1) Several factors may be programmed for inclusiveness. (2) Factors that do not contribute to that simulation assign zero (0) exponents. (3) Selecting exponents may be intriguing in the absence of data. Start with some guess of expected behavior and let the feedback from the material property behavior to guide the next update. (4) The exponents can also be evaluated if there is some data available especially from combined testing. This can be done by expressing the expanded equation using logs and then evaluate them by using the least squares method 
since the exponents will be the coefficients of linear algebraic equations. (5) To simulate block fatigue evaluate the degradation up to the end of the first block then use the degraded properties for the second block and repeat for additional blocks - degradation after second input to third etc. (6) Unloading generally requires different exponent then loading. Select the unloading exponent to simulate the expected loading-unloading by stress cycle. (7) Intermittent time effects are handled the same way as block fatigue. (8) Combined fatigue (thermo mechanical) is simulated by using the corresponding cycles and stresses in the respective factors. (9) Frequency effects are simulated in combination with cyclic loads. Stress value is not needed here since it is included in the cyclic effects factor. (10) The static stress and the temperature factors when present are used in combination with all active factors for that simulation effect. (11) To simulate fabrication process use the temperature factor, the stress factor due to pressure, a flow model and update the geometry through the finite element model of the component. The user will invent other ways to use MFIM that the author has not even thought of yet.

The most important point to remember is that MFIM is to be used incrementally where the current values are updated from global thermo structural, etc. analyses. This may require a two level iteration: (a) local to achieve local equilibrium. During the iteration, the factors are changed until local equilibrium is reached; and (b) global to achieve global equilibrium with respect to boundary loads and supports conditions and internal stress field or energy input in the increment equals the energy added to the structure. Another important point to note is that the exponent for specific factor in MFIM represents a general trend and not the entire precise path from its reference value to its final value. Also, note that the MFIM is used for all the properties that is the same factors but with different exponent. Results from sample cases for MFIM application are described for: metal matrix composites (MMC), and a nickel base alloy. As mentioned previously, these results are presented to illustrate a few of the generic features of the MFIM applicability to convey to the reader what has be done and infer therefrom what can be done. The specific how is described in the references.

\section{MFIM application to MMC}

In this sample case MFIM is part of the METCAN (Metal Matrix Composite Analyzer) [3]. The simulation for the properties to be shown starts from the fabrication process as depicted in Fig. 3.

The constituent material properties used are summarized in Table 1.

MMC Simulation of the transverse strength is shown in Fig. 4. For this simulation the factors for temperature, stress, and time were activated with respective exponents about 0.5 . It can be observed that the simulation predictions track the data almost exactly. That simulation is very complex because it incorporates matrix, interphase and fibers. It starts from the fabrication process and ends with monotonic loading to fracture. Sample simulation results for fatigue of the same composite are compared with data in Fig. 5. 
Step I Processing - Cool down from processing temperature $\left(T_{p}\right)$ to room temperature $\left(T_{0}\right)$

Step II Heat up to use temperature $\left(T_{u}\right)$ from room temperature

Step III Apply mechanical load to obtain stress-strain data
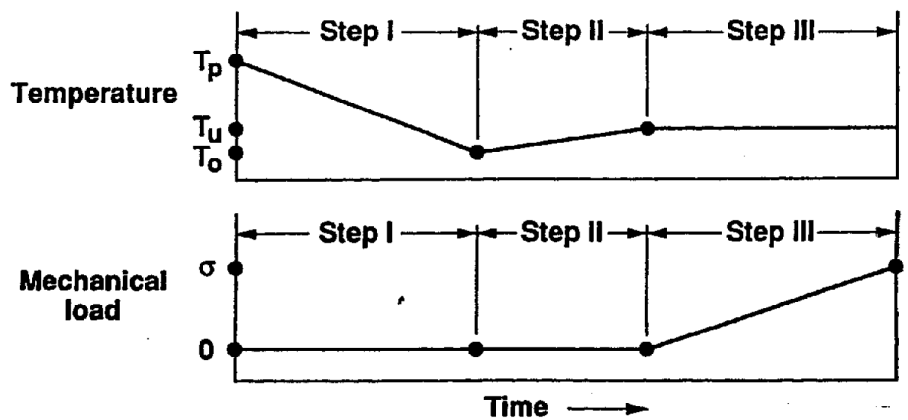

Figure 3: METCAN Metal Matrix Composite Behavior - computational simulation sequence.

Table 1: Constituent (fiber/matrix) material properties used in METCAN.

\begin{tabular}{|c|c|c|c|c|}
\hline & \multicolumn{4}{|c|}{ Material Properties } \\
\hline & \multicolumn{2}{|c|}{ Fiber } & \multicolumn{2}{|c|}{ Matrix } \\
\hline & Sic $^{a}$ & sic b $^{b}$ & $T-15-3^{a}$ & $T 1-6-4^{b}$ \\
\hline p, Ib/in. ${ }^{3}$ & 0.110 & 0.108 & 0.172 & 0.170 \\
\hline $\mathbf{T}_{\mathbf{m}},^{\circ} \mathbf{F}$ & 4870 & 4500 & 3000 & 3000 \\
\hline$E_{11}$, Mpsi & 62 & 60 & 12 & 17 \\
\hline$E_{22}, M p s i$ & 62 & 60 & 12 & 17 \\
\hline $\mathbf{G}_{12}$, Mpsi & 26 & 20 & 5 & 7 \\
\hline$v_{12}$ & 0.19 & 0.30 & 0.32 & 0.30 \\
\hline$\alpha_{11}, \mathrm{ppm} /{ }^{\circ} \mathrm{F}$ & 27 & 2.7 & 4.7 & 5.2 \\
\hline$\alpha_{22}, \mathrm{ppm} /{ }^{\circ} \mathrm{F}$ & 2.7 & 2.7 & 4.7 & 5.2 \\
\hline $\mathbf{s}_{11}, \mathbf{k s i}$ & 500 & 500 & 60 & 74 \\
\hline$S_{22}, \mathrm{ksi}$ & 500 & 500 & 60 & 74 \\
\hline $\mathrm{s}_{12}, \mathrm{ksl}$ & 300 & 250 & 36 & 44 \\
\hline
\end{tabular}

Private communication from "B. A. Lerch and 'P. R. Nimmer NASA Glenn Research Center

It is observed that the agreement is very good. In addition, the simulation identifies the types of failures that lead to specimen fracture that is achievable by the use of the MFIM. For this case, the mechanical cycle term in the MFIM was also activated with an exponent of about 0.5 for all the constituent properties. Simulation results for stress at times are compared in Fig. 6. As can be seen the agreement is very good. 


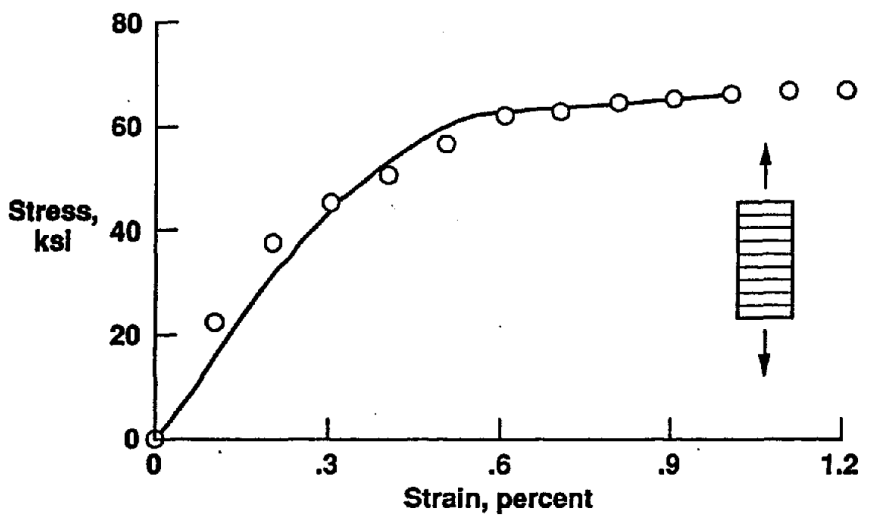

Figure 4: $\quad$ Transverse stress strain curve of $\mathrm{SiC} / \mathrm{Ti}-6-4 \mathrm{~T}=73^{\circ} \mathrm{F}, \mathrm{FVR}=0.34$.

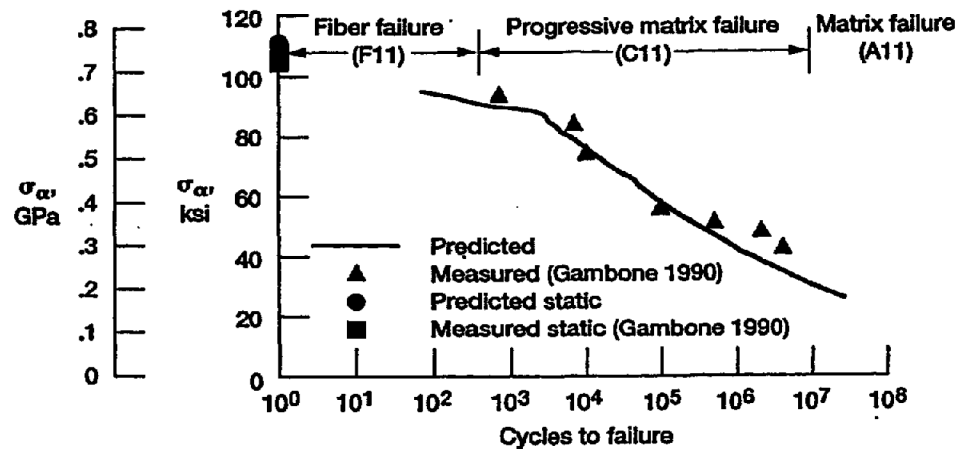

Figure 5: $\quad$ Predicted isothermal fatigue life [0] SCS6/Ti-24A1-11 Nb, 0.35 $\operatorname{FVR}\left(70^{\circ} \mathrm{F}\left(23^{\circ} \mathrm{C}\right), \mathrm{R}=0.1\right)$.

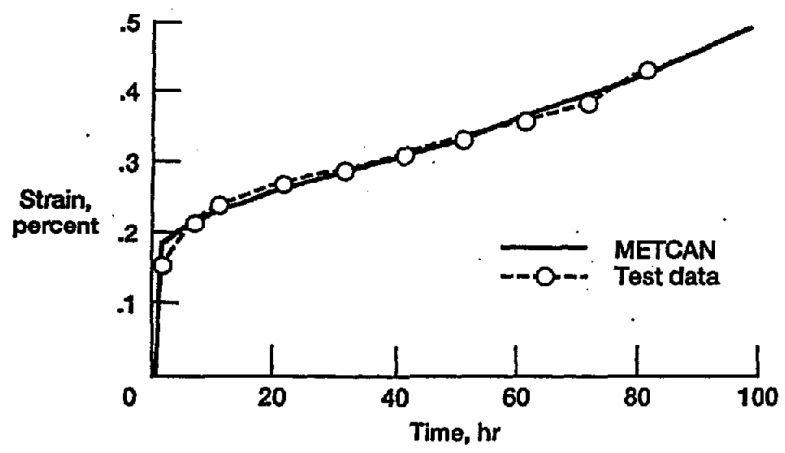

Figure 6: Creep behavior of [0] SCS6/Ti-24Al-11Nb composite at $815^{\circ} \mathrm{C}$ and $310 \mathrm{MPa} ; \mathrm{FVR}=0.35$; stress-free temperature $=815^{\circ} \mathrm{C}$. 


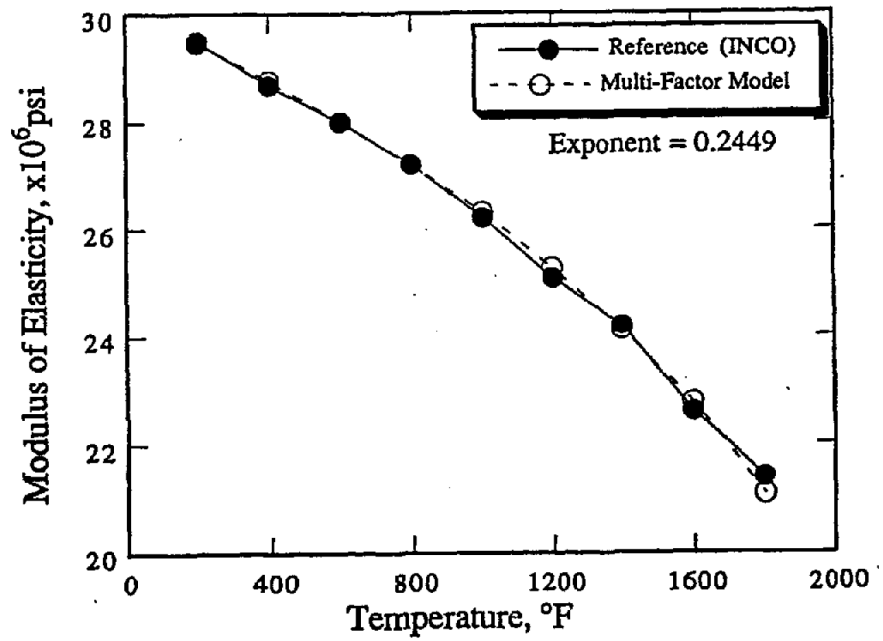

Figure 7: Calibration of nickel base alloy $713 \mathrm{C}$ elastic modulus as a function of temperature.

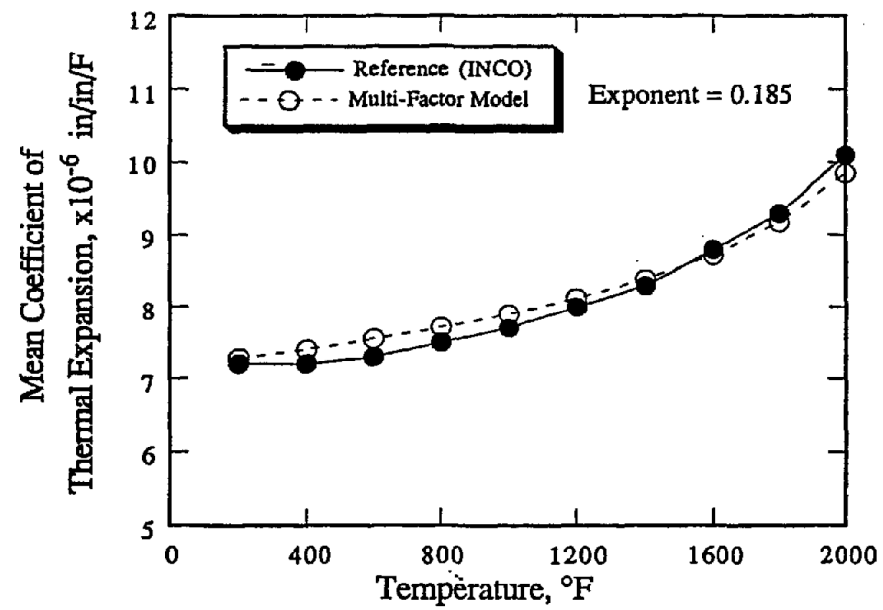

Figure 8: Calibration of nickel base alloy IN-100 coefficient of thermal expansion as a function of temperature.

The time factor was activated for the fabrication process and the tensile load to fracture. Additional simulation results are described in $[3,4]$. 


\section{Properties for nickel base alloy}

Simulation results of temperature effects on nickel base alloy 713C properties [5] are shown respectively in Fig. 7 for modulus, Fig. 8 for thermal expansion coefficient, Fig. 9 for thermal heat conductivity, and in Fig. 10 for heat capacity. Collectively the comparisons are in good agreement.

Collectively these results show the effectiveness of MFIM.

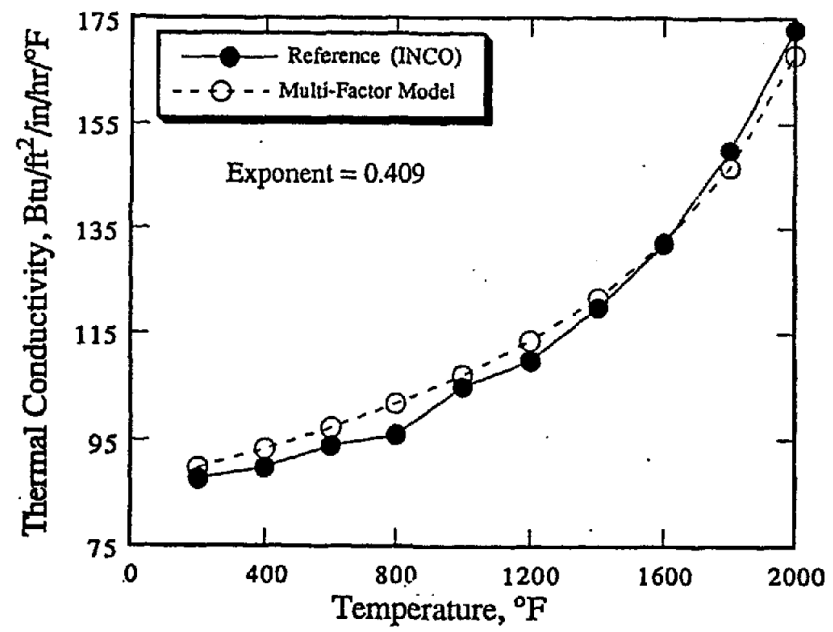

Figure 9: Calibration of nickel base alloy MAR-M 200 thermal conductivity as a function of temperature.

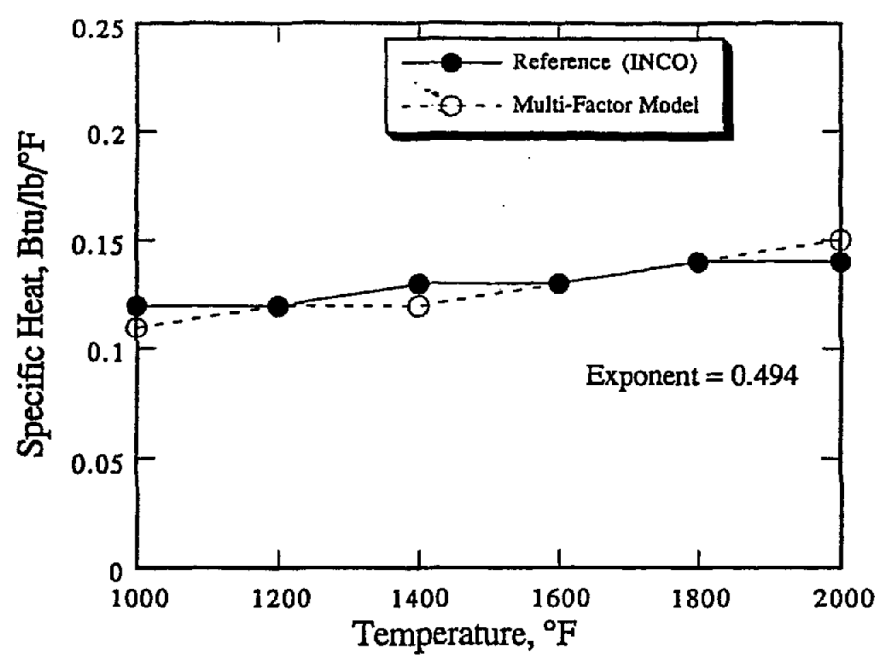

Figure 10: Calibration of nickel base alloy IN-100 specific heat as a function of temperature. 


\section{Concluding remarks}

The salient concluding remarks from a description of the multi-factor interaction model (MFIM) to predict complex material behavior by using a single equation and select applications are: (1) MFIM is a material point simulator. It is unique and inclusive for both composites and metals. (2) It is used incrementally in combination with analyses that provide local stress information. (3) It usually requires alternative solutions consisting of both local and global convergence. (4) Applications for meta matrix composites under different loading conditions predicted results that were in very good agreement with test data. (5) Familiarity and confidence and extensive usage will be gained by continuing usage application to complex solutions of material behavior and to simulate difficult and even untried problems.

\section{References}

[1] Chamis, C.C., Lark, R.F. and Sinclair, J.H.: Integrated theory for Predicting the Hydrothermal Mechanical Behavior of Composite Structural Components. ASTM pp. 160-192.

[2] Chamis, C.C. and Hopkins, D.A.: Thermo Viscoplastic Nonlinear Constitutive Relationships Structural Analysis of High Temperature Metal Matrix Component. NASA TM 87291, 1985

[3] Chamis, C.C., Murthy, P.L.N. and Hopkins, D.A.: Computational Simulation of High Temperature Metal Matrix Composites Cyclic Behavior. ASTM, STP 1080, 1990, pp 56-69.

[4] Tong, M.T., Singhal, S.N., Chamis, C.C. and Murthy, P.L.N.: Simulation of Fatigue Behavior of High Temperature Metal Matrix Composites. ASTM Reprint from Standard Technical Publication, 1253, 1996, pp. 540-551.

[5] Boyce, L. and Chamis, C.C.: Probabilistic Constitutive Relationships for Cyclic Material Strength Models. AIAA.ASME/ASCE/AHS $29^{\text {th }}$ Structures, Structural Dynamics and Materials Conference. Part 3, AIAA, 1988, pp. 1299-1306.

[6] Progressive Fracture Structural Analysis of National Wind Tunnel Structures by L. Minnetyan, NASA CR 198485, May 1996. 